\title{
Research Article \\ Reducing Unnecessary Brain Computed Tomography Scan in a Tertiary Center
}

\author{
Mohamed Abdoun ${ }^{1, *}, \mathbb{D}^{\mathbb{D}}$, Abdelmoneim Mahgoub $^{1}$, J. Motwali $^{2}$ \\ ${ }^{1}$ Department of Pediatric, Arryan Hospital, Dr Suliman Al Habib Group, Riyadh 11635, Saudi Arabia \\ ${ }^{2}$ College of Medicine, Red Sea University, Portsudan, Sudan
}

\section{ARTICLE INFO}

Article History

Received 22 June 2020

Accepted 28 August 2020

Keywords

Brain CT scan

meningitis

lumbar puncture

brain herniation

pediatric

\begin{abstract}
Brain Computed Tomography (CT) is routinely requested before Lumbar Puncture (LP) to rule out increased intracranial pressure. However, normal brain radiography does not abate the risk of herniation and unnecessarily delays the course of treatment. Thus, the primary aims of this study were to evaluate the frequency of brain CT scan abnormalities in children with suspected meningitis and to assess under what circumstances such changes are expected, with a secondary aim of reducing unnecessary brain CT scans. A retrospective study was conducted on 86 children with suspected meningitis before LP. Patients who underwent CT scans on suspicion of other neurological disorders were excluded. CT scan in $94.2 \%(n=81)$ cases were reported normal. $40 \%(n=2)$ cases with abnormal CT scan had altered sensorium as compared to $7.4 \%(n=6)$ with normal CT scan $(p=0.01)$. Furthermore, $46.5 \%$ of neuroimaging requests were not indicated. Herniation was not reported in our study. We conclude that indiscriminate brain CT scans have a limited role without clinical indications. In agreement with previous research, we recommend that requests for brain CT prior to LP be made on particular indications, as per clinical guidelines. Future prospective studies and re-auditing of practice continue to expand this body of evidence.
\end{abstract}

(c) 2020 Dr. Sulaiman Al Habib Medical Group. Publishing services by Atlantis Press International B.V. This is an open access article distributed under the CC BY-NC 4.0 license (http://creativecommons.org/licenses/by-nc/4.0/).

\section{INTRODUCTION}

Acute bacterial meningitis is a rapidly progressive disease that causes substantial morbidity and mortality [1]. The rationale behind diagnosis is the identification of the causative organism via Lumbar Puncture (LP) [2]. This is an invasive procedure used to remove Cerebrospinal Fluid (CSF) from the subarachnoid space [3]. In rare cases, however, the withdrawal process can hasten brain herniation because of Increased Intracranial Pressure (ICP) as well as the continuous leak of CSF through the needle opening into the subarachnoid membrane [3].

Clinicians routinely use brain Computed Tomography (CT) prior to LP to identify ICP. The indiscriminate use of imaging unnecessarily exposes patients to radiation and delays antibiotic initiation [4]. Furthermore, brain herniation has been reported in normal CT scans because of edema that cannot be detected radiologically [5].

A number of studies in accordance with guidelines has demonstrated that abnormal CT scans are rare in children without clinical signs of ICP [6,7]. These signs include disturbed consciousness, hemodynamic instability, fixed dilated pupils, focal neurological signs, or seizures. There is limited research in Saudi Arabia regarding the proportion of abnormal brain CT scans in children with suspected meningitis.

Corresponding author. Email: abdoonson111@gmail.com

Peer review under responsibility of the Dr. Sulaiman Al Habib Medical Group Data availability statement: The data that support the findings of this study are available from the corresponding author [M.A.], upon reasonable request.
The present study thus seeks to evaluate the frequency of CT scan abnormalities in children with suspected meningitis prior to LP as well as clinical predictors for such abnormalities. The secondary aim of this study is to reduce the number of unnecessary brain CT scans and, by extension, radiation exposure. This project, along with further research, will help us improve pediatric care.

\section{PATIENTS AND METHODS}

The present study was conducted in the pediatrics department at Arryan Hospital/Dr Sulaiman Al-Habib Medical Group (HMG) in Riyadh, Saudi Arabia. HMG is a private tertiary center and a Joint Commission International-accredited hospital. The study was approved by the HMG Institutional Review Board (Approval No: RC19.04.31).

This retrospective study aims to evaluate the frequency of CT abnormalities in children with suspected meningitis prior to LP and to identify clinical predictors of such CT scan abnormalities, with a secondary aim of reducing unnecessary brain CT scans and radiation exposure. Our inclusion criteria were patients of both sexes aged 2-15 years with suspected meningitis prior to LP. The diagnosis of meningitis is based on the patient's clinical presentation, which includes fever, headache, lethargy, neck stiffness, altered mental status, and nonblanchable rashes [8]. Our exclusion criteria were patients younger than 2 years, those with comorbidities (e.g., immunodeficiency), and those who underwent CT scans on suspicion of other neurological disorders. 
The data were obtained from an electronic hospital record system (Volunteers for Intercultural and Definitive Adventures) and classified on a Microsoft Office Excel 2010 spreadsheet (Microsoft Corporation, Redmond, WA, USA). Our parameters included age, sex, CT scan results, and clinical indications from the imaging request. The results of $\mathrm{CT}$ scans according to the interpretation of the radiologist were divided into two groups: Group I, representing normal CT scans, and Group II, representing abnormal CT scans (e.g., focal abnormalities or brain edema). Data were displayed as descriptive statistics, with mean \pm Standard Deviation (SD) for continuous data and number/percentage for categorical data. Statistical analysis was performed using SPSS version 19 (IBM Corporation, Armonk, NY, USA). Student $t$-test and Chi-square test were used to measure continuous and categorical data, respectively. Logistic regression analysis was incorporated to estimate the impact of risk factors on abnormal CT scan result. Statistical significance was measured with a $p$-value $<0.05$.

Lastly, clinical indications from imaging were audited based on recommendations provided by the American Academy of Pediatrics (AAP) and National Institute of Health and Care Excellence (NICE). Clinical indications include disturbed consciousness, hemodynamic instability, fixed dilated pupils, focal neurological signs, and seizures.

\section{RESULTS}

\subsection{Description of the Study Population}

The records of a total of 93 patients with suspected meningitis were initially considered during the study period. Among these, seven patients were excluded. A total of 86 patients met the necessary inclusion criteria and were recruited for the study (Figure 1).

The age of participants ranged from 2 to 15 years, with a mean of $7.31 \pm 3.58$ years and a sex frequency of $65.1 \%(56 / 86)$ male and $34.9 \%(30 / 86)$ female. Most patients presented with seizures (43\%) and lethargy (23.1\%). Baseline clinical characteristics and CT scan findings are shown in Table 1.

\subsection{Classification of Brain CT Findings}

The CT scans have been split up according to radiological findings. Normal brain imaging includes non-pathological brain changes or those with brain atrophies.

Abnormal CT scans have brain edema, meningeal enhancement, or focal lesions such as abscess, hemorrhage, or mass effect. The distribution of the patients according to brain CT scan findings is illustrated in Table 2.

\subsection{Clinical Predictors of CT Scan Abnormalities}

Logistic regression was incorporated to adjust for different confounders. Altered mental status was the only significant predictor of brain CT scan abnormalities in our study (Table 3).

Table 1 Demographic and clinical variables based on CT scan results

\begin{tabular}{lccc}
\hline Variables & Normal CT scan & Abnormal CT scan & $p$ \\
\hline Age (mean \pm SD) & $7.22 \pm 3.61$ & $8.80 \pm 2.77$ & $0.3^{\mathrm{a}}$ \\
Sex & & & \\
$\quad$ Male & 52 & 4 & $0.34^{\mathrm{b}}$ \\
$\quad$ Female & 29 & 1 & \\
Altered mental status & 6 & 2 & $0.01^{\mathrm{b}}$ \\
Seizures & 36 & 1 & $0.8^{\mathrm{b}}$ \\
Focal neurological signs & 3 & 0 & $0.6^{\mathrm{b}}$ \\
Lethargy & 18 & 2 & $0.09^{\mathrm{b}}$ \\
Fever & $38.3 \pm 0.75$ & $38.5 \pm 0.70$ & $0.83^{\mathrm{a}}$ \\
Photophobia & 14 & 2 & $0.2^{\mathrm{b}}$ \\
Vomiting & 23 & 3 & $0.13^{\mathrm{b}}$ \\
Neck stiffness & 17 & 2 & $0.32^{\mathrm{b}}$ \\
WBC & $10.07 \pm 7.29$ & $10.50 \pm 7.77$ & $0.82^{\mathrm{a}}$ \\
Neutrophils & $6.53 \pm 5.89$ & $7.15 \pm 8.27$ & $0.49^{\mathrm{a}}$ \\
CRP & $32.33 \pm 58.9$ & $80.7 \pm 107.2$ & $0.2^{\mathrm{a}}$ \\
\hline
\end{tabular}

${ }^{\mathrm{a}}$ Independent $t$-test. ${ }^{\mathrm{b}} \mathrm{Chi}$-square test and Fisher's exact test were used, as appropriate, to calculate. $p<0.05$; the level of significance was fixed at $<0.05$. CRP, C-reactive protein; WBC, white blood cell count.

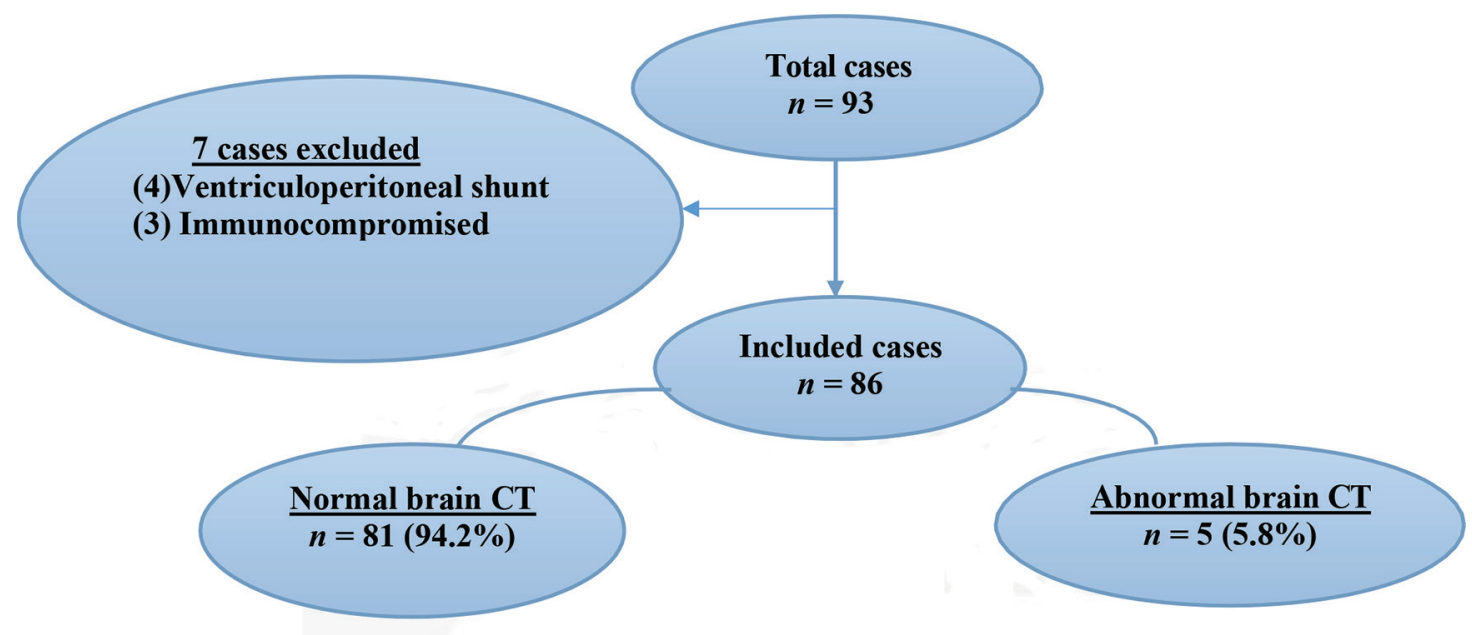

Figure 1 Classification of study population. 
Table 2 Result of brain computed tomography

(CT) scans in children suspected with meningitis

\begin{tabular}{lc}
\hline Result of CT scan & $\boldsymbol{n}(\%)$ \\
\hline Normal & $81(94.2)$ \\
Abnormal & \\
$\quad$ Brain edema & $4(12)$ \\
Brain abscess & $1(12)$ \\
\hline
\end{tabular}

Table 3 Clinical predictors of abnormal brain CT scans

\begin{tabular}{llll}
\hline Variables & $\boldsymbol{B}$ & $\mathrm{SE}$ & $\boldsymbol{p}$ \\
\hline Age & 0.07 & 0.07 & 0.34 \\
Lethargy & 0.11 & 0.58 & 0.06 \\
Vomiting & 1.3 & 0.94 & 0.15 \\
Photophobia & 1.16 & 0.95 & 0.22 \\
Neck stiffness & 0.92 & 0.95 & 0.33 \\
Alter mental status & 0.211 & 0.086 & 0.016 \\
Neutrophil & 0.017 & 0.116 & 0.88 \\
CRP & 0.009 & 0.009 & 0.31 \\
\hline
\end{tabular}

$B$, beta coefficient; CRP, C-reactive protein; SE, standard error.

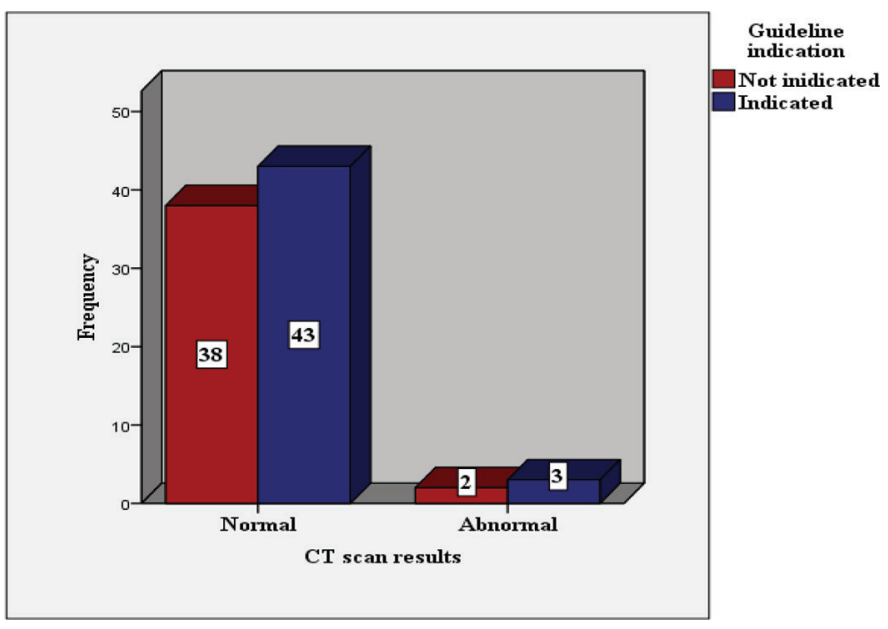

Figure 2 Frequency of patients that had brain CT scans as per $\mathrm{AAP} / \mathrm{NICE}$ indication.

\subsection{Indications of Brain CT Scans Prior to LP}

The study revealed that 46 (53.5\%) of the brain CT scans performed were indicated according to AAP/NICE guidelines, with a mean of $6.77 \pm 3.25$ years. Forty (46.5\%) scans, which constitute the rest of the brain CT scans, were not indicated. Of the patients who underwent LP, none had a brain herniation. The frequency of CT scan abnormalities based on clinical indication is illustrated in Figure 2.

\section{DISCUSSION}

The trend of performing brain imaging prior to LP is on the rise, even in patients with no clinical signs. The information available in pediatric care is limited. Thus, this study intends to provide an analytic overview of brain CT scans performed prior to LP in children with suspected meningitis. Demographic variables among the study group were statically nonsignificant.

The indiscriminate use of CT scans in children provides limited information. Our study demonstrated that the vast majority of scans performed were normal or unchanged (94.2\%) and that the observed frequency of abnormal scans prior to LP was low (5.8\%). Several studies have concordant results. For instance, the Vancouver study described normal brain CT scans for 41 patients screened prospectively [9]. Similarly, Seleem et al. [10] reported a small number of abnormalities (0.06\%) for 101 patients assessed.

Meningitis is associated with a variable degree of brain edema that is either mild in the absence of ICP signs or is not detected radiologically [5]. Clinicians are thus advised to consider clinical assessment as a baseline prior to LP and to reserve imaging for those indicated. Accordingly, our study found two patients with brain edema who underwent LP without complications in the absence of ICP signs. Hasbun et al. [11] similarly recommended clinical features to identify those who are unlikely to develop brain herniation.

We analyzed the following clinical variables to determine their relationship to brain CT scan abnormalities: age, sex, new-onset seizures, focal neurological deficits, and altered consciousness. No significant demographic differences were seen in our data. Seizures were the most commonly observed findings in our patients; however, they did not signify abnormal brain CT scans. In contrast, Chen et al. [12] reported notable neuroimaging abnormalities in $61(26 \%)$ patients with seizures. A transient increase in ICP within $30 \mathrm{~min}$ of a seizure is a well-documented phenomenon; LP is therefore contraindicated during this time [12]. Altered consciousness was found to be significantly associated with brain CT scan abnormalities in our study. Correspondingly, Vafaie et al. [13] demonstrated a vital relationship between CT changes and decreased levels of consciousness. In a prospective study of 113 patients, Gopal et al. [14] likewise concluded that altered mental status strongly predicted CT scan abnormalities.

Finally, as part of a quality improvement project, we reviewed the practice of requesting CT scans and whether it has met with clinical guidelines issued by the AAP and NICE. The study revealed that $46.5 \%$ of CT scans were not indicated. Of these, two displayed image abnormalities; however, none had brain herniation after puncture. Studies revealed significant association between the estimated radiation doses provided by cerebral CT scans and the subsequent incidence of leukemia, brain tumors, and rate of cataract formation [15]. Therefore, it is advised that LP safety be assessed based on clinical decision rather than a radiological one. Blood culture and antibiotics should not be delayed if a CT scan is required [7].

Nonetheless, these findings have to be seen in light of several limitations. The study was dependent on the availability of welldocumented and detailed notes. Inaccuracy of information could have led to the underestimation of CT scan indications. Future prospective studies with larger sample sizes are thus recommended.

\section{CONCLUSION}

The majority of brain CT scans in children with suspected meningitis prior to LP were normal, and altered sensorium 
was significantly correlated with CT brain abnormalities. Indiscriminate brain imaging is thus not advisable. The study recommends guideline adherence to select those most likely to benefit from brain imaging. Further prospective studies are advisable to expand this body of evidence.

\section{CONFLICTS OF INTEREST}

The authors declare they have no conflicts of interest.

\section{AUTHORS' CONTRIBUTION}

AM, MA contributed in conceptualization, designing and writing the manuscript. JM contributed in data processing and review the edited manuscript. MA contributed in formal analysis and writing the original draft. AM supervised the project. However, all the authors review the manuscript and approve the final draft of the manuscript.

\section{ACKNOWLEDGMENTS}

The authors are grateful to Dr Hussein Al Hajji (consultant pediatric neurologist) for revising the scientific component of the article.

\section{REFERENCES}

[1] Daoud AS, Omari H, Al-Sheyyab M, Abuekteish F. Indications and benefits of computed tomography in childhood bacterial meningitis. J Trop Pediatr 1998;44;167-9.

[2] Steigbigel NH. Computed tomography of the head before a lumbar puncture in suspected meningitis - is it helpful? N Engl J Med 2001;345;1768-70.

[3] Baker ND, Kharazi H, Laurent L, Walker AT, Williamson DS, Weissman BN, et al. The efficacy of routine head computed tomography (CT scan) prior to lumbar puncture in the emergency department. J Emerg Med 1994;12;597-601.

[4] Smith-Bindman R, Lipson J, Marcus R, Kim KP, Mahesh M, Gould R, et al. Radiation dose associated with common computed tomography examinations and the associated lifetime attributable risk of cancer. Arch Intern Med 2009;169;2078-86.

[5] Joffe AR. Lumbar puncture and brain herniation in acute bacterial meningitis: a review. J Intensive Care Med 2007;22;194-207.

[6] Swanson D. Meningitis. Pediatr Rev 2015;36;514-26.

[7] National Institute for Health and Care Excellence. Meningitis (bacterial) and meningococcal septicaemia in under 16s. Available from: https://www.nice.org.uk/guidance/cg102/resources/meningitisbacterial-and-meningococcal-septicaemia-in-under-16srecognition-diagnosis-and-management-pdf-351093 25611205.

[8] Centers for Disease Control and Prevention. Meningitis. 2019. Available from: https://www.cdc.gov/meningitis/bacterial.html.

[9] van Crevel H, Hijdra A, de Gans J. Lumbar puncture and the risk of herniation: when should we first perform CT? J Neurol 2002;249;129-37.

[10] Seleem W, Elsotouhy A, Farooq A, Sharaf N, Badran H, Shoaebi $\mathrm{M}$, et al. Computed tomography of the head prior to lumber puncture in suspected cases of meningitis in children, in Qatar. Acad J Pediatr Neonatol 2017;6;555732.

[11] Hasbun R, Abrahams J, Jekel J, Quagliarello VJ. Computed tomography of the head before lumbar puncture in adults with suspected meningitis. N Engl J Med 2001;345;1727-33.

[12] Chen CY, Chang YJ, Wu HP. New-onset seizures in pediatric emergency. Pediatr Neonatol 2010;51;103-11.

[13] Vafaie M, Mirzarahimi M, Enteshari-Moghaddam A, Mousavi S. Evaluation of brain CT-scan results and indications before lumbar puncture in children suspected of meningitis hospitalized in Ardabil city hospital. Int Surg J 2018;5;3585-8.

[14] Gopal AK, Whitehouse JD, Simel DL, Corey GR. Cranial computed tomography before lumbar puncture: a prospective clinical evaluation. Arch Intern Med 1999;159;2681-5.

[15] Pearce MS, Salotti JA, Little MP, McHugh K, Lee C, Kim KP, et al. Radiation exposure from CT scans in childhood and subsequent risk of leukaemia and brain tumours: a retrospective cohort study. Lancet 2012;380;499-505. 